\title{
AN ELEMENTARY INEQUALITY IN FUNCTION THEORY
}

\author{
by W. N. EVERITT
}

(Received 18 September, 1968)

1. In the theory of self-adjoint operators in Hilbert space and of formally self-adjoint linear differential equations there are many situations involving analytic functions on the complex plane whose singularities are confined to the real axis and where the growth of the function at such singular points is strictly limited.

In this note we give an inequality for such functions which is useful in the neighbourhood of points on the real axis. There is also an application to an elementary proof of a known result concerning the bound of the resolvent of a self-adjoint operator in Hilbert space.

2. $C$ denotes the complex plane, $R$ the real line and $R_{+}$the non-negative real numbers.

Let $f(\cdot)$ be analytic on the complex plane $C$ and regular at least in the half-planes $\operatorname{im} z>0$ and $\operatorname{im} z<0$ with $z=x+i y$; thus the singularities of $f(\cdot)$ are confined to the real axis although the function may be regular at some real points.

Suppose that there is a real number $M$ such that $0<M<\infty$ and

$$
|f(z)| \leqq \frac{M}{|y|}, \text { for all } z \in C \text { with } y=\operatorname{im} z \neq 0 .
$$

Let $D$ be the domain of $C$ in which $f(\cdot)$ is regular and put $F=C-D=D^{c}$; thus $D$ is an open set of the plane and $F$ is a closed subset of the real line. An argument based on the maximum modulus theorem shows that (1) implies that $f(\cdot)$ cannot be an integral function on $C$ unless it is the null function; for this argument see Section 3 below. Thus, taking $f(\cdot)$ not to be null, it follows that the set $F$ cannot be the empty set $\phi$.

Let the distance mapping $d(\cdot)$ be defined by $d(\cdot): D \rightarrow R_{+}$with

$$
d(z)=\inf \{|z-w|: w \in F\}, \quad \text { for all } z \in D .
$$

Since $F$ is closed it follows that

$$
0<d(z)<\infty, \quad \text { for all } z \in D .
$$

THEOREM 1. Let $f(\cdot), D$ and $F$ be defined as above and suppose the inequality (1) to be satisfied;

then

$$
|f(z)| \leqq \frac{2 M}{d(z)}, \quad \text { for all } z \in D,
$$

and this result is best possible. 
If, in addition, the set $F$ is bounded below (above), say $b=\inf \{F\}>-\infty$ (say $b=\sup \{F\}<\infty)$, then

$$
|f(z)| \leqq \frac{M}{d(z)}
$$

for all $z \in D$ with $\operatorname{re} z<b(\operatorname{re} z>b)$; this result is also best possible.

Note. The inequality (4) is best seen as a bound for the values of $f(\cdot)$ on or near the real axis, particularly when $f(\cdot)$ has points of regularity on the axis. For large values of $y=\operatorname{im} z$, it is clear that (1) is a better inequality than (4), but that, if $\xi \in D \cap R$, then (4) gives a better bound for $|f(z)|$ when $z$ is in the neighbourhood of $\xi$ than (1) would give. On the other hand (5) is, for the half-plane, a better inequality than (1).

Proof. Let $z$ be any point of $D$. Without loss of generality we can suppose that $\mathrm{re} z=0$, since the inequality (1) is invariant under translation along the real axis; i.e., if $\xi \in R$, then

$$
|f(z+\xi)| \leqq \frac{M}{|y|}, \quad \text { for all } z \in C \text { with } \operatorname{im} z \neq 0 .
$$

Thus it is sufficient to prove the inequality (4) for any point on the imaginary axis including the origin if that point should belong to $D$.

Suppose firstly that $0 \in F$. Then (1) implies (4) for all points $i y$ on the imaginary axis with $y \neq 0$; for in this case the origin is the nearest point of $F$ and $d(i y)=|y|$, so that, from (1),

$$
|f(i y)| \leqq \frac{M}{|y|}<\frac{2 M}{|y|}=\frac{2 M}{d(i y)} .
$$

Now suppose that $0 \in D$. Then since $D$ is open there is a positive number $p$ such that the open interval $(-p, p) \subset D$ but at least one of the points $\pm p$ belongs to $F$. If $|y| \geqq p / \sqrt{ } 3$, then $d(i y) \leqq 2|y|$ and, again from (1),

$$
|f(i y)| \leqq \frac{M}{|y|} \leqq \frac{2 M}{d(i y)}, \quad|y| \geqq \frac{p}{\sqrt{3}},
$$

which is the required result for this range of $y$. Now let the number $r$ satisfy $0<r<p$ and consider the function $g_{r}(\cdot)$ defined by $g_{r}(z)=\left(z^{2}-r^{2}\right) f(z)$ for $|z|<p$. Clearly $g_{r}(\cdot)$ is regular in this domain and so, by the maximum modulus theorem (see [5], Section 5.1),

$$
\left|g_{r}(z)\right| \leqq \sup \left\{\left|r^{2} e^{2 i \theta}-r^{2}\right| \cdot\left|f\left(r e^{i \theta}\right)\right|: 0 \leqq \theta \leqq 2 \pi\right\}
$$

for $|z| \leqq r . \quad$ A calculation shows that $\left|e^{2 i \theta}-1\right|=2|\sin \theta|$ and from (1) we obtain

$$
\left|\operatorname{im}\left\{r e^{i \theta}\right\} . f\left(r e^{i \theta}\right)\right| \leqq M \quad(0 \leqq \theta \leqq 2 \pi) .
$$

$\dagger$ The use of this function is suggested by the proof of Lemma 2.11 of [4]. 
Thus (8) now gives

$$
\begin{aligned}
\left|g_{r}(z)\right| & \leqq \sup \left\{2 r^{2}\left|\sin \theta \cdot f\left(r e^{i \theta}\right)\right|: 0 \leqq \theta \leqq 2 \pi\right\} \\
& \leqq \sup \left\{2 r\left|\operatorname{im}\left(r e^{i \theta}\right) \cdot f\left(r e^{i \theta}\right)\right|: 0 \leqq \theta \leqq 2 \pi\right\} \\
& \leqq 2 M r \quad \text { for } \quad|z| \leqq r
\end{aligned}
$$

and so

$$
|f(z)| \leqq \frac{2 M r}{\left|z^{2}-r^{2}\right|} \text { for } \quad|z|<r .
$$

Since this last result is true for all $r<p$, let $r \rightarrow p-0$ to give

$$
|f(z)| \leqq \frac{2 M p}{\left|z^{2}-p^{2}\right|} \text { for } \quad|z|<p .
$$

In (9) put $z=i y$ with $|y|<p$ to obtain

$$
\begin{aligned}
|f(i y)| \leqq \frac{2 M p}{y^{2}+p^{2}} & =\frac{p}{\left(y^{2}+p^{2}\right)^{\frac{1}{2}}} \frac{2 M}{\left(y^{2}+p^{2}\right)^{\frac{1}{2}}} \\
& \leqq \frac{2 M}{d(i y)} \quad \text { for } \quad|y|<p .
\end{aligned}
$$

Together (7) and (10) prove the inequality (4) in the case when $0 \in D$.

The inequality (4) is now seen to be true for all $z \in D$.

It may be seen that this inequality is best possible by considering the example

$$
f(z)=\left(z^{2}-p^{2}\right)^{-1} \quad \text { for all } z \in C \text { with } z \neq \pm p,
$$

where $p$ is some positive number. A calculation shows that $\left|\operatorname{im} z \cdot\left(z^{2}-p^{2}\right)^{-1}\right| \leqq(2 p)^{-1}$ for all $z \in C$ with $z \neq \pm p$, so that this example satisfies the hypothesis (1) with $M=(2 p)^{-1}$. Now

$$
|f(0)|=\frac{1}{p^{2}}=\frac{2 M}{p}=\frac{2 M}{d(0)}
$$

which is a case of equality in (4). Actually the derivation of (10) above shows that equality in (4) can only occur at points on the real axis.

Now consider the second inequality (5) and suppose that $F$ is bounded below; the proof when $F$ is bounded above is similar.

Without loss of generality we can consider the case when $b$, the lower bound of $F$, is zero (translating the origin if necessary), i.e. $0 \in F$ but $x \in D$ if $x<0$.

Let $p>0$ and consider the domain of $C$ determined by $|z+p|<p$; using the same argument above which gave the inequality (9) we see that

$$
|f(z)| \leqq \frac{2 M p}{\left|(z+p)^{2}-p^{2}\right|}=\frac{2 M p}{\left|z^{2}+2 p z\right|} \text { for } \quad|z+p|<p
$$


and this is valid for all $p>0$ since $f(\cdot)$ is regular for the half-plane re $z<0$. Now let $p \rightarrow \infty$ in this last result to give

$$
|f(z)| \leqq \frac{M}{|z|}=\frac{M}{d(z)} \quad \text { for all } z \text { with re } z<0,
$$

since for points with re $z<0$ it is clear, from the definition in (2), that $d(z)=|z|$; this establishes the inequality (5).

It may be seen that the inequality (11) is best possible on considering the simple example $f(z)=z^{-1}$ on $C$.

3. Note that if the inequality (1) holds not over the whole plane $C$ but only in the domain $\{z:|z-p|<r\}$, where $p, r \in R$ and $r>0$, then $F$ and $D$ as subsets of this domain can be defined as before; in this case the inequality (4) can be reformulated.

If $f(\cdot)$ satisfies (1) over $C$ and is also an integral function, then the inequality (9) holds for all $p>0$; letting $p \rightarrow \infty$ shows that $f(\cdot)$ must be the null function. Thus if $f(\cdot)$ is not null, the set $F$ defined in Section 2 cannot be the empty set $\phi$.

4. Let $H$ be a complex Hilbert space with $I$ the identity operator and let $T$, with domain $D(T)$, be a self-adjoint operator in $H$ (see [1], Section 41). Let $C$ be the complex plane and write $\lambda=\mu+i v$.

The resolvent set $\rho(T)$ of $T$ is the set of all points of the complex plane $C$ for which the inverse operator $(T-\lambda I)^{-1}$ exists, is bounded and defined on the whole space $H$; the spectrum $\sigma(T)$ of the operator $T$ is the complement of $\rho(T)$ in $C$. It is known that $\rho(T)$ is an open set of $C$ and $\sigma(T)$ is closed and is a subset of the real line $R$. For these definitions and results see [1], Section 43.

If we put $R_{\lambda}(T)=(T-\lambda I)^{-1}$, then this operator is defined for $\lambda \in \rho(T)$ and has the following properties:

$$
\begin{gathered}
\left\|R_{\lambda}(T)\right\| \leqq|v|^{-1} \quad \text { for } \lambda \in C \text { with } v=\operatorname{im} \lambda \neq 0, \\
R_{\lambda}(T)^{*}=R_{\lambda}(T) \text { for all } \lambda \in \rho(T),
\end{gathered}
$$

where * denotes the adjoint operator; for (1) see [1], Theorem 3 of Section 43 and for (2) see [1], Section 44. Also for any elements $f, g \in H$ the function $\left(R_{\lambda}(T) f, g\right)$, defined from the inner product on $H$, is an analytic function of $\lambda$ regular in the set $\rho(T)$; see [1], Section 65 .

For any $\lambda \in \rho(T)$ define the distance function $d(\cdot, \sigma(T))$ on $\rho(T)$ by

$$
d(\lambda, \sigma(T))=\inf \{|\lambda-\zeta|: \zeta \in \sigma(T)\}
$$

so that $0<d(\lambda, \sigma(T))<\infty$, for all $\lambda \in \rho(T)$.

THEOREM 2. Let $T, \rho(T)$ and $d(\cdot, \sigma(T))$ be defined as above; then

$$
\left\|R_{\lambda}(T)\right\|=\{d(\lambda, \sigma(T))\}^{-1} \quad \text { for all } \lambda \in \rho(T) .
$$

Note. For a proof of this Theorem depending on the spectral radius of $R_{\lambda}(T)$ see [3], Chapter V, Section 3.5. 
Proof. If $T$ is self-adjoint, then for all real $\mu$ the operator $T-\mu I$ on the domain $D(T)$ is also self-adjoint; thus we can, without loss of generality, prove (4) in the case re $\lambda=0$ and it will then be true in general, i.e. it is sufficient to prove that (4) holds on the imaginary axis.

Suppose firstly that $0 \in \sigma(T)$. Then from (3) we have $d(i v, \sigma(T))=|v|$ and in view of (1) above it is sufficient to prove that $\left\|R_{i v}(T)\right\|<|v|^{-1}$ is impossible. Suppose to the contrary that $\left\|R_{i v}(T)\right\|=(|v|+\varepsilon)^{-1}$, where $\varepsilon>0$. Then we have $(|v|+\varepsilon)\left\|R_{i v}(T) g\right\| \leqq\|g\|$, for all $g \in H$. Given any $f \in D(T)$, define $g=(T-i v l) f$ to give

$$
\begin{aligned}
(|v|+\varepsilon)^{2}\|f\|^{2} & =(|v|+\varepsilon)^{2}\left\|R_{i v}(T)(T-i v I) f\right\|^{2} \\
& =(|v|+\varepsilon)^{2}\left\|R_{i v}(T) g\right\|^{2} \\
& \leqq\|g\|^{2}=\|(T-i v I) f\|^{2},
\end{aligned}
$$

valid for all $f \in D(T)$. Now by expressing $\|\cdot\|^{2}$ as the inner product (,) we have (see also [1], Section 43, proof of Theorem 3), for all $f \in D(T)$,

$$
\|(T-i v I) f\|^{2}=\|T f\|^{2}+v^{2}\|f\|^{2} .
$$

Together (5) and (6) give

$$
\begin{aligned}
&\|T f\|^{2} \geqq\left\{(|v|+\varepsilon)^{2}-|v|^{2}\right\}\|f\|^{2} \\
&>\varepsilon^{2}\|f\|^{2}, \quad \text { for all } f \in D(T) .
\end{aligned}
$$

However, as in the proof of [1], Section 43, Theorem 4, this last result shows that $T$ is regular at 0 , i.e. that $0 \in \rho(T)$. This contradiction shows that $\left\|R_{i v}(T)\right\|=|v|^{-1}$ and (4) is then established in this case.

Suppose secondly that $0 \in \rho(T)$. Then since this set is open, there is $p>0$ such that the open internal $(-p, p) \subset \rho(T)$ but at least one of the points $\pm p$ belongs to $\sigma(T)$. Assume for the moment that the theorem is true for $R_{0}(T)$, i.e. that

$$
\left\|R_{0}(T)\right\|=\frac{1}{d(0, \sigma(T))}=\frac{1}{p},
$$

which will be established later; then, as above, $p^{2}\left\|R_{0}(T) g\right\|^{2} \leqq\|g\|^{2}$ for all $g \in H$ and this implies that $p^{2}\|f\|^{2} \leqq\|T f\|^{2}$, for all $f \in D(T)$. This gives from (6) above

$$
\begin{aligned}
\|(T-i v I) f\|^{2} & =\|T f\|^{2}+v^{2}\|f\|^{2} \\
& \geqq\left(p^{2}+v^{2}\right)\|f\|^{2},
\end{aligned}
$$

for all $f \in D(T)$. Since $T$ is regular at $i v$ the range of $T-i v I$ is $H$ and so this last result may be recast as

$$
\|g\|^{2} \geqq\left(p^{2}+v^{2}\right)\left\|R_{i v}(T) g\right\|^{2} \quad \text { for all } g \in H
$$

and so

$$
\left\|R_{i v}(T)\right\| \leqq\left(p^{2}+v^{2}\right)^{-\frac{1}{2}}=\{d(i v, \sigma(T))\}^{-1}
$$


If, for $v \neq 0$, we had strict inequality in this last result, i.e. $\left\|R_{i v}(T)\right\|=\left\{\left(p^{2}+v^{2}\right)^{+1}+\varepsilon\right\}^{-1}$ for some $\varepsilon>0$, then

$$
\left\{\left(p^{2}+v^{2}\right)^{\frac{1}{2}}+\varepsilon\right\}^{2}\|f\|^{2} \leqq\|(T-i v I) f\|^{2}=\|T f\|^{2}+v^{2}\|f\|^{2}
$$

or

$$
\left[\left\{\left(p^{2}+v^{2}\right)^{\frac{1}{2}}+\varepsilon\right\}^{2}-v^{2}\right]^{\frac{1}{2}}\|f\| \leqq\|T f\|=\|(T \pm p I) f \mp p f\| \leqq\|(T \pm p I) f\|+p\|f\| ;
$$

i.e.

$$
\left(\left[\left\{\left(p^{2}+v^{2}\right)^{\frac{1}{2}}+\varepsilon\right\}^{2}-v^{2}\right]^{\frac{1}{2}}-p\right)\|f\| \leqq\|(T \pm p I) f\|,
$$

which is valid for all $f \in D(T)$. This last expression yields $\|(T \pm p I) f\|>\varepsilon\|f\|$, which, as before, would imply that $T$ is regular at both points $\pm p$, i.e. that $\pm p$ both belong to $\rho(T)$. This contradiction establishes equality in (9).

Thus it remains to prove (8). The inequality $\left\|R_{0}(T)\right\| \geqq p^{-1}$ may be established as above by assuming the contrary, which then implies that $T$ is regular at both points $\pm p$. Thus (8) will be established if we can prove $\left\|R_{0}(T)\right\| \leqq p^{-1}$ and for this we use the inequality (5) of Theorem 1.

Consider the operator $T^{2}$ defined by $D\left(T^{2}\right)=\{f \in D(T) ; T f \in D(T)\}$ and then $T^{2} f=T(T f)$ for all $f \in D\left(T^{2}\right)$. It is known that $T^{2}$ is self-adjoint (see [1], Section 46, Theorem 2) and the spectrum of $T^{2}$ is determined by $\sigma\left(T^{2}\right)=\left\{p^{2} ; p \in \sigma(T)\right\}$. In the case of $T$ above this implies that $p^{2} \in \sigma\left(T^{2}\right)$ but $\left(-\infty, p^{2}\right) \subset \rho\left(T^{2}\right)$.

Let $f, g \in H$. Consider the function $\phi(\cdot)$ defined by

$$
\phi(\lambda)=\left(R_{\lambda}\left(T^{2}\right) f, g\right), \quad \text { for all } \lambda \in \rho(T) .
$$

Then $\phi(\cdot)$ is analytic on $C$ and regular on $\rho\left(T^{2}\right)$.

We have

$$
\begin{aligned}
|\phi(\lambda)| & \leqq\left\|R_{\lambda}\left(T^{2}\right) f\right\|\|g\| \\
& \leqq\left\|R_{\lambda}\left(T^{2}\right)\right\|\|f\|\|g\| \\
& \leqq|\nu|^{-1}\|f\|\|g\|, \quad \text { for all } \lambda \in C \text { with } \operatorname{im} \lambda \neq 0,
\end{aligned}
$$

on using (1) of this section. Thus $\phi(\cdot)$ satisfies the conditions of Theorem 1 , with

$$
M=\|f\|\|g\|, \quad D=\rho\left(T^{2}\right), \quad F=\sigma\left(T^{2}\right)
$$

and $F$ is bounded below by $p^{2}$; in this case $d(\lambda)=d\left(\lambda, \sigma\left(T^{2}\right)\right)$. From (5) of Theorem 1 we thus obtain

$$
|\phi(\lambda)| \leqq\left\{d\left(\lambda, \sigma\left(T^{2}\right)\right)\right\}^{-1}\|f\|\|g\|, \quad \text { for all } \lambda \text { with re } \lambda<p^{2},
$$

and so in particular

$$
|\phi(0)| \leqq p^{-2}\|f\|\|g\|
$$


In this last result put $g=R_{0}(T) f$, as we may do since $f$ and $g$ are arbitrary, to give

$$
\begin{aligned}
\left|\left(R_{0}\left(T^{2}\right) f, R_{0}\left(T^{2}\right) f\right)\right| & =\left\|R_{0}\left(T^{2}\right) f\right\|^{2} \\
& =|\phi(0)| \\
& \leqq p^{-2}\|f\|\left\|R_{0}\left(T^{2}\right) f\right\|,
\end{aligned}
$$

i.e.

$$
\left\|R_{0}\left(T^{2}\right) f\right\| \leqq p^{-2}\|f\|, \quad \text { for all } f \in H
$$

i.e.

$$
\left\|R_{0}\left(T^{2}\right)\right\| \leqq p^{-2}
$$

Now it is not difficult to see that $R_{0}\left(T^{2}\right)=R_{0}(T)^{2}$ and so from (2) above that

$$
R_{0}\left(T^{2}\right)=R_{0}(T) R_{0}(T)^{*} \text {. }
$$

From [2], Section 22, Theorem 4 we have $\left\|R_{0}\left(T^{2}\right)\right\|=\left\|R_{0}(T) R_{0}(T)^{*}\right\|=\left\|R_{0}(T)\right\|^{2}$, so that (10) implies

$$
\left\|R_{0}(T)\right\|^{2} \leqq p^{-2} \quad \text { or } \quad\left\|R_{0}(T)\right\| \leqq p^{-1} .
$$

This completes the proof of the theorem.

5. The referee has pointed out that there is some overlap between the result given above in Section 4 and the paper by R. G. Bartle entitled "Spectral localization of operators in Banach spaces ", Math. Annalen 153 (1964), 261-9. See in particular pp. 261-5.

\section{REFERENCES}

1. N. I. Akhiezer and I. M. Glazman, Theory of linear operators in Hilbert space, Volumes I and II (New York, 1961).

2. P. R. Halmos, Introduction to Hllbert space (New York, 1951).

3. T. Kato, Perturbation theory for linear operators (Berlin, 1966).

4. E. C. Titchmarsh, Eigenfunction expansions associated with second order differential equations, Part I, 2nd edition (Oxford, 1962).

5. E. C. Titchmarsh, Theory of functions, 2nd edition (Oxford, 1939).

\section{UNIVERSITY OF DUNDEE}

